\title{
Motion onset captures attention: A rejoinder to Franconeri and Simons (2005)
}

\author{
RICHARD A. ABRAMS and SHAWN E. CHRIST \\ Washington University, St. Louis, Missouri
}

\begin{abstract}
Recently, we have provided evidence that the onset of motion captures attention (Abrams \& Christ, 2003, 2005a, 2005b). In the present article, we clarify the motion onset hypothesis, we discuss recent data (Franconeri \& Simons, 2005) that, at least on the surface, seem to challenge the hypothesis, and we present results from a new experiment (Christ \& Abrams, 2005). Finally, we conclude that, although motion onset does indeed appear to capture attention, motion in the absence of a motion onset might also attract attention under certain circumstances.
\end{abstract}

In an attempt to explain the capture of attention by a number of different dynamic events under a single theoretical umbrella, Franconeri and Simons (2003) introduced the behavioral urgency hypothesis:

The behavioral urgency hypothesis predicts capture [of attention] only by stimuli that indicate the potential need for immediate action. New objects, objects that move suddenly, and looming objects are all behaviorally urgent, and all strongly capture attention. Relative to these stimuli, receding objects and uniquely colored items are not as likely to require immediate action, and they do not strongly capture [attention]. (p. 1008)

Commenting on this work, we (Abrams \& Christ, 2005) pursued the possibility that the hypothesis might involve a degree of circularity (i.e., Dynamic events that are behaviorally urgent capture attention. How does one know whether a dynamic event is behaviorally urgent? If it captures attention.), and we presented results showing that an object that appeared to recede could indeed capture attention if the receding motion was rendered with stereo depth cues (unlike the stimuli used by Franconeri \& Simons, 2003). Those results contradict the findings reported by Franconeri and Simons (2003) and, more importantly, are inconsistent with their behavioral urgency hypothesis. In addition, we noted that our proposed motion onset hypothesis (Abrams \& Christ, 2003) could accommodate the attentional advantage afforded to the moving stimuli employed in several of the Franconeri and Simons (2003) conditions. $^{1}$

Most recently, Franconeri and Simons (2005) have presented a reply to our comment. In their reply, Franconeri and Simons (2005) present data from a new experiment that they argue are inconsistent with the motion onset hypothesis. Importantly, the version of the motion onset hypothesis

Correspondence concerning this article should be addressed to R. A. Abrams, Department of Psychology, Washington University, St. Louis, MO 63130 (e-mail: rabrams@wustl.edu). tested by Franconeri and Simons (2005) differs in critical ways from our motion onset hypothesis as we have previously described it (Abrams \& Christ, 2003, 2005a, 2005b). Furthermore, the data presented as evidence against the hypothesis are, in fact, entirely consistent with the hypothesis. Nevertheless, additional data from their new experiment not reported in their reply but provided in a personal communication (August 2, 2005) may indeed require that the hypothesis be tempered somewhat. Thus, the two main goals of the present article are (1) to reiterate and clarify the motion onset hypothesis and (2) to discuss the experimental results reported by Franconeri and Simons (2005) within the context of the hypothesis. To accomplish the second goal, we also report data from a new experiment.

\section{The Motion Onset Hypothesis}

Our motion onset hypothesis states that the onset of motion in a previously existing static object will capture attention (Abrams \& Christ, 2003, 2005a, 2005b). The hypothesis stemmed from our research findings in which elements that underwent a motion onset were processed preferentially relative to elements that had been continuously moving, had been stationary, or had undergone a motion offset (Abrams \& Christ, 2003). Our finding of no attentional benefit for continuous motion is consistent with findings of other researchers who failed to find an attentional advantage for objects that were already moving when they first appeared (e.g., Hillstrom \& Yantis, 1994; Yantis \& Egeth, 1999). One reason for this might be the attenuated response to motion that is observed within the first few hundred milliseconds after the appearance of a new object (Torriente, Valdes-Sosa, Ramirez, \& Bobes, 1999), as we noted in our 2003 article.

What is it that constitutes "motion"? In contrast to the claims of Franconeri and Simons, we do not believe that "motion" is synonymous with "a change at a luminance edge" (Franconeri \& Simons, 2005, p. 963). As we stated recently, we believe instead "that attention is attracted not by low-level changes in luminance-defined contours, such as that which occurs when an object moves and also when 
an object shrinks, but instead by a change in the perceived location of the object" (Abrams \& Christ, 2005b, p. 222). That is, we think the relevant motion onset is one that can be conceptualized at a somewhat higher level than mere onset of motion on the retina: It might be regarded as the onset of object motion. We identified several sources of evidence that suggest a distinction between the perceived motion of an object and the actual retinal motion of the object's contours. ${ }^{2}$ It is the former type of motion whose onset we believe to be important, as we have previously indicated. Importantly, we believe that attentional capture by motion onset does not require the presence of any specific low-level visual event. Instead, if the object is perceived to have started to move, it will capture attention. This principle is in complete accord with the results that Franconeri and Simons (2005) report in their comment, a topic to which we turn next.

\section{Experimental Results of Franconeri and Simons (2005)}

In the Franconeri and Simons (2005) experiment, subjects viewed a display containing three or five items. Then, while the subjects were looking away from the display briefly $(>800 \mathrm{msec})$, the initial display was replaced by a second one that contained a moving item that was often in the same location as a previously stationary item. Coincident with the return of their gaze to the display, subjects completed a search task. The results were clear: The item that was now moving (which was likely to have replaced a stationary item from the prior display) attracted attention. However, in contrast to Franconeri and Simons's (2005) conclusion, that result is entirely consistent with the motion onset hypothesis: The object that started to move attracted attention. The fact that subjects were prevented from witnessing the motion onset at the time at which it occurred is not relevant to an evaluation of the hypothesis. Presumably, subjects encoded the objects in the scene, and then a short time later, they learned that one previously static object was now moving. This caused attention to be directed to the object that had begun to move, resulting in a performance advantage in the experiment. Indeed, the Franconeri and Simons (2005) result provides good support for our hypothesis and provides a useful control to rule out the possibility that the effect is due merely to changes at luminance edges.

\section{Additional Results From the Franconeri and Simons (2005) Experiment}

In a personal communication (August 2, 2005), Franconeri and Simons reported an additional analysis from their experiment that had not been included in their reply. Specifically, they compared trials on which the motion onset element appeared in a place previously occupied by a placeholder and trials on which the motion onset element appeared in a previously unoccupied location. This comparison was possible because the display size varied from trial to trial in their experiment: Any mismatch from one trial to the next meant that some elements would be appearing in previously unoccupied locations. Importantly, the comparison revealed no difference between these two types of trials. In other words, motion onset captured attention equally well when a previously existing object had begun to move while subjects were looking away and when a potentially new object was in motion when it was first gazed upon. This result, on the surface, does appear to challenge our suggestion that motion onset must occur in a preexisting object in order to capture attention. Nevertheless, there are certain aspects of the Franconeri and Simons (2005) experiment that may limit the significance of the finding. In particular, the search elements in their experiment were arranged along an imaginary circle with an element always appearing at the 12 o'clock position regardless of display size and with two or four other elements appearing at other locations (spaced equally around the circle). When the display changed from one display size to another (while subjects were looking away), it is possible that subjects perceived the unique elements in the second display (i.e., the elements at locations other than 12 o'clock) to be new objects, as Franconeri and Simons (personal communication, August 2, 2005) have assumed. In that case, the Franconeri and Simons results would be evidence for an advantage to motion without motion onset of a preexisting object. It is also possible that the subjects perceived most of the search elements to be old objects even when the display changed from one display size to the other. This is possible because the distance between search elements in the two display sizes was very small. In particular, the two unique elements under Display Size 3 were each only $0.26^{\circ}$ from an element in the Display Size 5 condition (the letters themselves were $1.26^{\circ}$ tall). In that case, the subjects may indeed have perceived the moving element to be a preexisting object that was previously stationary. In order to arrive at a definitive answer, more work will be needed with stimuli that do not have this limitation.

Indeed, we (Christ \& Abrams, 2005) have recently completed an experiment that corroborates the conclusions suggested by Franconeri and Simons's (personal communication, August 2, 2005) new analysis. In our experiment, subjects first viewed a display containing figure-eight placeholders. Then, the placeholders changed into letters, and subjects searched the display for one of two target letters. Coincident with the presentation of the search display, one of the letters also started to move (similar to the approach that we have used previously; e.g., in Abrams $\&$ Christ, 2003). We directly manipulated the age of the search array elements by varying the duration of the placeholder display: On some trials, the placeholders were visible for $1 \mathrm{sec}$ before changing into letters (coincident with the change to letters, one element began to move). On other trials, placeholders were not presented - only the search array appeared, with one element that was moving. In our experiment, because of the relatively long intertrial interval $(3,000 \mathrm{msec})$, there was no ambiguity regarding the extent to which the search elements were new or old objects. Consistent with the new analysis of Franconeri and Simons (personal communication, August 2, 2005), we did find a search advantage for the moving element 
relative to static elements under conditions in which the elements appeared in a previously unoccupied location (the advantage was $45 \mathrm{msec}$ ). It thus appears to be the case that motion is capable of capturing attention in the absence of a motion onset - as Franconeri and Simons (2005) have argued. Nevertheless, in our experiment, we also found a considerable additional advantage to motion onset when it occurred in a preexisting object (the advantage was $97 \mathrm{msec}$ ). Thus, it also is clear that motion onset of a preexisting object can provide a substantial additional attentional advantage. Mean reaction times for the experiment are shown in Figure 1. ${ }^{3}$

It is important to note that our findings, as well as those from Franconeri and Simons's (personal communication, August 2, 2005) analysis, appear to be inconsistent with results reported by Folk, Remington, and Wright (1994), Hillstrom and Yantis (1994), and Yantis and Egeth (1999). These researchers all failed to find an advantage for a moving object when the elements in the display were new objects. More work will be needed in order to understand the basis of these differences.

\section{Earlier Results From Franconeri and Simons (2003)}

Some readers may wonder about one unexplained feature of Franconeri and Simons's (2003) results. In that study, attention was captured by objects in which looming was simulated by size changes, whereas attention was not captured by objects in which size changes simulated recession. As noted earlier, we showed that the onset of receding movement can indeed attract attention when the motion in depth is rendered with stereo depth cues (Abrams \& Christ, 2005b). However, it is not clear why attention was captured by expanding objects but not by shrinking ones in the Franconeri and Simons (2003) study. Given the differences in results obtained with shrinking objects (in Franconeri \& Simons, 2003) compared with stereorendered recession (in Abrams \& Christ, 2005b), we think

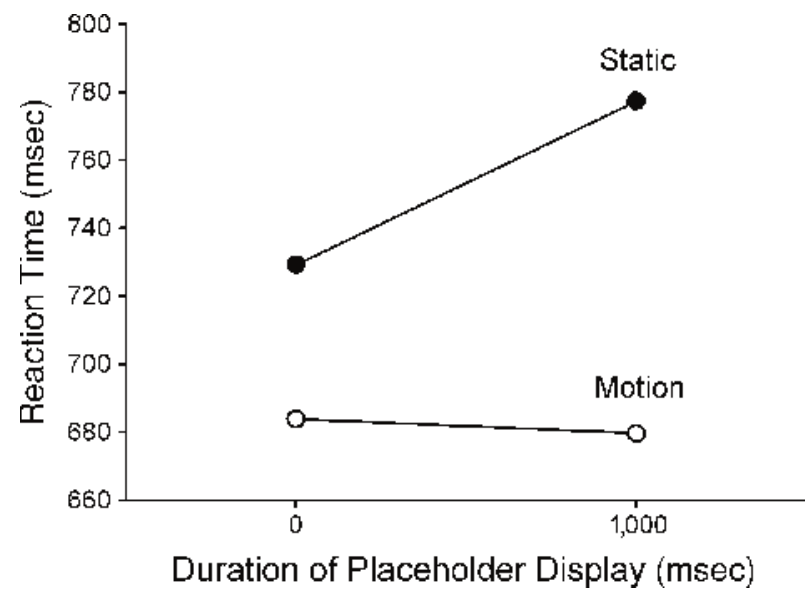

Figure 1. Mean reaction times for target identification shown separately for each movement condition (motion or static) and each duration of the placeholder display ( 0 or $1,000 \mathrm{msec}$ ). the most prudent explanation is that size changes may not be an effective way of simulating motion in depth.

\section{Conclusions}

Is an onset of motion necessary in order for motion to capture attention? It does not appear to be. Does motion onset provide a substantial additional benefit? It does. Why might motion onset capture attention? As we have suggested previously, the onset of motion is a powerful cue to animacy - that the object in question may be alive. The ability to detect nearby living things may yield a substantial survival benefit. Such a benefit could be of use whether or not the observer witnessed the motion onsetif something is moving now that was not moving before, it could be alive, and that might be important.

\section{REFERENCES}

Abrams, R. A., \& Christ, S. E. (2003). Motion onset captures attention. Psychological Science, 14, 427-432.

Abrams, R. A., \& Christ, S. E. (2005a). Onset but not offset of irrelevant motion disrupts inhibition of return. Perception \& Psychophysics, 67, 1460-1467.

Abrams, R. A., \& Christ, S. E. (2005b). The onset of receding motion captures attention: Comment on Franconeri and Simons (2003). Perception \& Psychophysics, 67, 219-223.

Christ, S. E., \& Abrams, R. A. (2005). [Attention to old and new objects]. Unpublished raw data.

Folk, C. L., Remington, R. W., \& Wright, J. H. (1994). The structure of attentional control: Contingent attentional capture by apparent motion, abrupt onset, and color. Journal of Experimental Psychology: Human Perception \& Performance, 20, 317-329.

Franconeri, S. L., \& Simons, D. J. (2003). Moving and looming stimuli capture attention. Perception \& Psychophysics, 65, 999-1010.

Franconeri, S. L., \& Simons, D. J. (2005). The dynamic events that capture visual attention: A reply to Abrams \& Christ (2005). Perception \& Psychophysics, 67, 962-966.

Hillstrom, A. P., \& Yantis, S. (1994). Visual motion and attentional capture. Perception \& Psychophysics, 55, 399-411.

Masson, G. S., \& Stone, L. S. (2002). From following edges to pursuing objects. Journal of Neurophysiology, 88, 2869-2873.

Sugihara, H., Murakami, I., Shenoy, K. V., Andersen, R. A., \& Komatsu, H. (2002). Response of MSTd neurons to simulated 3D orientation of rotating planes. Journal of Neurophysiology, 87, 273-285.

Torriente, I., Valdes-Sosa, M., Ramirez, D., \& Bobes, M. A. (1999). Visual evoked potentials related to motion-onset are modulated by attention. Vision Research, 39, 4122-4139.

YANTIS, S., \& EgETH, H. E. (1999). On the distinction between visual salience and stimulus-driven attentional capture. Journal of Experimental Psychology: Human Perception \& Performance, 25, 661-676.

\section{NOTES}

1. Regarding our interpretation of our results, Franconeri and Simons (2005, p. 963) made the following erroneous statement: "On the basis of this evidence that receding motion captures attention, they [Abrams \& Christ, 2005b] argued that the motion onset hypothesis can explain all evidence in the literature for attention capture by dynamic events." In fact, what we stated was "It is now possible to interpret their [Franconeri \& Simons's, 2003] results, our earlier results (Abrams \& Christ, 2003), and the results of researchers such as Folk et al. (1994), Hillstrom and Yantis (1994), and Yantis and Egeth (1999) with a single, simple explanation: The onset of motion of preexisting objects captures attention. ..." (Abrams \& Christ, 2005b, p. 222).

2. In Abrams and Christ (2005b), we stated:

For example, cells in a brain area sensitive to movement (the medial superior temporal area) respond selectively not merely to local components of the 
stimulus motion, but also to a higher order, derived representation of the orientation of the depicted object (Sugihara, Murakami, Shenoy, Andersen, \& Komatsu, 2002). Also, Masson and Stone (2002) showed that pursuit eye movements are not driven by the retinal motion of the contours of the pursued object, but instead by a computed representation of the movement of the object itself. (p. 222)

3. The data were analyzed using a 2 (movement condition: target moving or static) $\times 2$ (duration of placeholder display: 0 or $1,000 \mathrm{msec}$ ) repeated measures ANOVA. There was a significant main effect of movement condition $[F(1,10)=85.28, p<.001]$ and an interaction between movement condition and the duration of the placeholder display
$[F(1,10)=21.69, p<.005]$. There was not a main effect for the duration of the placeholder display. Note that one limitation of this experiment is that the distractors may have been more distracting with the $0-\mathrm{msec}$ placeholder display relative to the 1,000 -msec placeholder display because of the greater luminance transients associated with the appearance of the objects, although the magnitude of the contribution of this factor is not known.

(Manuscript received July 28, 2005;

revision accepted for publication December 5, 2005.) 